\title{
Análisis de la calidad de vida del paciente en prediálisis y su relación con la dependencia para las actividades instrumentales de la vida diaria
}

\author{
Rosa María González-Tamajón ${ }^{1}$, Carmen Jiménez-Prietoํㅜㄹ ${ }^{1}$ Nazaret Campillo-Cañete ${ }^{1}$, María Victoria Gómez- \\ López ${ }^{2}$, Rodolfo Crespo-Montero ${ }^{1,2,3}$ \\ ${ }^{1}$ Departamento de Enfermería. Facultad de Medicina y Enfermería. Universidad de Córdoba. Córdoba. España \\ 2 Servicio de Nefrología. Hospital Universitario Reina Sofía. Córdoba. España \\ ${ }^{3}$ Instituto Maimónides de Investigación Biomédica de Córdoba. Córdoba. España
}

Como citar este artículo:

González-Tamajón RM, Jiménez-Prieto C, Campillo-Cañete N, Gómez-López MV, Crespo-Montero R. Análisis de la calidad de vida del paciente en prediálisis y su relación con la dependencia para las actividades instrumentales de la vida diaria.

Enferm Nefrol. 2020 0ct-Dic;23(4):361-370

\section{Resumen}

Objetivos: Analizar la calidad de vida relacionada con la salud de los pacientes con enfermedad renal crónica avanzada y determinar su relación con la dependencia para las actividades instrumentales de la vida diaria.

Material y Método: Se realizó un estudio descriptivo transversal en el Servicio de Nefrología del Hospital Universitario Reina Sofía de Córdoba, en el que se incluyeron pacientes con enfermedad renal crónica avanzada en estadios 4-5. Se utilizaron los siguientes cuestionarios: KDQOL-SF, para analizar la calidad de vida relacionada con la salud; Lawton y Brody, para dependencia para las actividades instrumentales de la vida diaria.

Resultados: Se estudiaron 105 pacientes con una edad de 67,8 $\pm 15,7$ años; 35 mujeres (33,3\%). En el KDQ0L-SF, las dimensiones más afectadas fueron los Cambios en el estado de salud, Situación laboral, Carga de la enfermedad renal, Salud general, Vitalidad y Rol físico. El 57,1\% de la muestra tenían algún grado de dependencia para las actividades instrumentales de la vida diaria. Los pacientes con mayor grado de dependencia presentaron peores puntuaciones en las dimensiones Efectos de la

\section{Correspondencia:}

Rosa María González Tamajón

Email: rosamglezt@gmail.com enfermedad renal, Situación laboral, Función cognitiva, Sueño, Función física, Rol físico, Dolor, Función social y Vitalidad.

Conclusiones: Los pacientes en prediálisis tienen disminuida la calidad de vida relacionada con la salud en las dimensiones Cambios en el estado de salud, Situación laboral, Carga de la enfermedad renal, Salud general, Vitalidad y Rol físico. Más de la mitad de la muestra presentan algún tipo grado de dependencia para la realización de las actividades instrumentales de la vida diaria.

PALABRAS CLAVE: calidad de vida relacionada con la salud; ERCA; prediálisis; actividades instrumentales de la vida diaria; actividades básicas de la vida diaria.

\section{Analysis of the quality of life of the predialysis patient and the relationship with the dependency for the instrumental activities of daily living}

\section{Abstract}

Objectives: To analyze the health-related quality of life (HRQoL) in patients with advanced chronic kidney disease in pre-dialysis and the relationship with the dependency for the instrumental activities of daily living (IADL). 
Material and Method: A descriptive and cross-sectional study was carried out in the Nephrology Service of the Reina Sofía University Hospital in Córdoba. It was a non-probability accidental sampling. The following questionnaires were used: KDQOL-SF to analyze HRQoL and Lawton y Brody for IADL dependency.

Results: 105 patients on pre-dialysis were studied with an age of $67,8 \pm 15,7$ years and 35 women $(33,3 \%)$. With regards to the $\mathrm{KDQOL}$, the most affected dimensions were Changes in health status, Work situation, Burden of kidney disease, General health, Vitality and Physical role. $57.1 \%$ of the participants had some degree of dependence for the instrumental activities of daily life. Patients with a higher degree of dependency had worse scores in the dimensions Effects of kidney disease, Work situation, Cognitive function, Sleep, Physical function, Physical role, Pain, Social function and Vitality.

Conclusions: Pre-dialysis patients have a decreased health-related quality of life in the dimensions Changes in health status, Work situation, Burden of kidney disease, General health, Vitality and Physical role. More than half of the participants show some type of dependency to carry out the instrumental activities of daily life.

KEYWORDS: health-related quality of life; ACKD; pre-dialysis; instrumental activities of daily living; basic activities of daily living.

\section{Introducción}

A medida que progresa la enfermedad renal crónica avanzada (ERCA) y el filtrado glomerular (FG) desciende por debajo de $30 \mathrm{ml} / \mathrm{min} / 1,73 \mathrm{~m}^{2}$, comienzan a aparecer los síntomas característicos de los estadios 4-5 de esta enfermedad. En su estadio final, cuando el riñón es incapaz de mantener la función renal compatible con la vida, se hace necesario una terapia renal sustitutiva (TRS)1-3.

La prevalencia de la ERCA ha ido en aumento en los últimos años ${ }^{4}$ y como consecuencia la incidencia y prevalencia de pacientes con ERCA en TRS, también ha ido incrementándose. Según el informe de diálisis y trasplante de 2018 de la Sociedad Española de Nefrología 5 , la incidencia fue de 147,3 pacientes por millón de población estando la prevalencia en 1.321,9 pacientes por millón de población. Este aumento en la incidencia y prevalencia es debido a diferentes factores, entre los que se puede destacar el progresivo envejecimiento de la población, el aumento de diabetes mellitus tipo 2 y síndrome metabólico, además de una mejora en el tratamiento de enfermedades crónicas que permiten mayor esperanza de vida ${ }^{6}$. Además de las alteraciones propias de la ERCA, la enfermedad va a limitar de manera significativa la calidad de vida de los pacientes que la padecen, desde los estadios iniciales hasta la etapa final, lo cual se va a ver reflejado en la vida diaria de estos pacientes ${ }^{7}$.

La calidad de vida relacionada con la salud (CVRS), es un concepto multidimensional que ha sido definido como la evaluación subjetiva que realiza un individuo sobre el impacto de la enfermedad y su tratamiento sobre la dimensión física, psicológica y social, valorando el impacto sobre la funcionalidad y el bienestar ${ }^{8}, \mathrm{y}$ existen múltiples estudios que analizan esta variable en los pacientes con ERCA en TRS; observándose que la gran mayoría de estos pacientes presentan síntomas emocionales como son la ansiedad y la depresión, los cuales han sido asociados a mayor cantidad de síntomas somáticos 9 . Sin embargo, el número de estudios que analicen la CVRS en pacientes con ERCA en la etapa prediálisis son escasos ${ }^{10}$.

Una de las variables que puede influir directamente en la CVRS es el grado de dependencia funcional, que puede afectar a la capacidad para llevar a cabo las actividades básicas de la vida diaria (ABVD) y las actividades instrumentales de la vida diaria (AIVD), que son aquellas que requieren mayor autonomía personal e interacción con el entorno, como ir de compras, preparar la comida, manejar dinero, usar medios de transporte... ${ }^{11}$. Las AIVD presentan déficits funcionales más precoces que las $A B V D$; sin embargo, la mayoría de los estudios realizados se centran en las $A B V D^{12}$. En este sentido, algunos estudios han explorado estas variables en pacientes en hemodiálisis, donde se observa que estos pacientes son más dependientes para las $A I V D$ que para las $A B V D$, repercutiendo ambas sobre la CVRS $^{13}$. Sin embargo, no existe mucha bibliografía en los estadios 4-5 de la ERCA o etapa de prediálisis.

Desde el punto de vista asistencial, es importante conocer el nivel de dependencia de estos pacientes para así poder intervenir. Según algunos estudios publicados en nuestro país, se ha podido constatar que la intervención del personal de enfermería en las consultas de ERCA es una labor fundamental ante la enfermedad, ya que supone una mejora del nivel de conocimiento para el paciente, para su bienestar y autocuidado, mejorando la adherencia al tratamiento y disminuyen- 
do la ansiedad del mismo, lo cual puede influir en una mejor calidad de vida gracias a una atención integra$\mathrm{da}$, individualizada y especializada en las consultas de ERCA $^{14,15}$.

Por tanto, dada la importancia que tiene la percepción de la CVRS en el paciente con ERCA, y su posible relación con la alteración de las AIVD, creemos que es importante el estudio de estas variables ante la escasa bibliografía encontrada al respecto ${ }^{16,17}$.

Por todo ello, el objetivo del presente estudio ha sido analizar la CVRS de los pacientes con ERCA en prediálisis y determinar su relación con la dependencia para las AIVD.

\section{Material y Método}

\section{Ámbito del estudio}

El estudio se llevó a cabo en el Servicio de Nefrología del Hospital Universitario Reina Sofía de Córdoba, en la consulta de Bajo Aclaramiento Renal, durante los meses de enero a marzo de 2020 .

\section{Diseño}

Se realizó un estudio observacional descriptivo de corte transversal.

\section{Población y muestra}

La población de estudio han sido las personas con ERCA en estadios 4-5, sin alteraciones cognitivas que le impidieran responder a los cuestionarios, atendidas en la consulta de Bajo Aclaramiento (Prediálisis) del Servicio Nefrología, siendo criterio de exclusión aquellos pacientes en tratamiento conservador (no entran en programa de TRS). El muestreo utilizado fue no probabilístico accidental, de todos los pacientes que acudieron a consulta durante el periodo que duró el estudio.

Aunque el tiempo de reclutamiento de la muestra programado en principio fue de enero hasta abril, a principios de marzo se dejó de recopilar datos debido a la pandemia por COVID-19.

\section{Variables del estudio}

Las variables de resultado fueron la CVRS y dependencia para las AIVD. Se estudiaron además otras variables como la dependencia para las ABVD, edad, sexo, FG, Índice de comorbilidad, Índice de Masa Corporal (IMC) y nivel de hemoglobina sérica ( $\mathrm{Hgb}$ ).

\section{Instrumentos de medida}

Para determinar la CVRS se utilizó el cuestionario validado KDQOL-SF, que es una adaptación del cuestionario SF-36 para pacientes con ERCA.

Para analizar la dependencia para las AIVD se empleó la escala de Lawton y Brody. Se trata de un cuestionario que consta de 8 ítems: capacidad de usar el teléfono, ir de compras, preparación de la comida, cuidado de la casa, lavado de la ropa, uso de medios de transporte, responsabilidad respecto a medicación y manejo de asuntos económicos. Las respuestas de cada ítem tienen un valor de 0 (dependiente) o 1 (independiente). La puntuación final consiste en la suma de los valores de esas respuestas y va desde 0 (máxima dependencia) a 8 (independencia total).

Para evaluar la dependencia para las ABVD, se empleó el test de Barthel, y para el análisis de la comorbilidad se utilizó el Índice de Comorbilidad de Charlson (ICC). El resto de variables fueron extraídas de la base de datos Servicio de Nefrología. Se dividió a los pacientes en estadio $4\left(F G>15 \mathrm{ml} / \mathrm{min} / 1,73 \mathrm{~m}^{2}\right)$ y estadio 5 $\left(F G<15 \mathrm{ml} / \mathrm{min} / 1,73 \mathrm{~m}^{2}\right)$.

\section{Recogida de datos}

Los datos se obtuvieron en la propia consulta de Bajo Aclaramiento de la UGC Nefrología, según la agenda de citación de dicha consulta. Los cuestionarios se cumplimentaron durante el proceso de acto único de la citada consulta, por lo que no hubo necesidad de añadir tiempo adicional de los pacientes, al que rutinariamente permanecen en la consulta. Los cuestionarios fueron administrados por una única profesional a aquellos pacientes que, tras ser informados del estudio a realizar y previa lectura del consentimiento informado, aceptaron colaborar en este estudio.

\section{Análisis estadístico}

Para las variables cualitativas se utilizó una distribución de frecuencias, mientras que para las variables numéricas se usó la media \pm la desviación estándar de la media, o la mediana y el rango intercuartílico, según siguieron o no una distribución normal. Para establecer comparaciones entre ambos sexos, se utilizó la $t$ de Student para muestras independientes o la $U$ de Mann Whitney, según siguieron o no una distribución normal. Se realizaron correlaciones con el test de Pearson para establecer asociaciones entre las diferentes variables. Se aceptó significación estadística para $p<0,05$. El análisis estadístico se realizó con el paquete estadístico SPSS 15.0 para Windows. 


\section{Aspectos éticos y legales}

La información obtenida ha sido tratada conforme a la actual Ley Orgánica 3/2018, de 5 de diciembre, de "Protección de Datos Personales y garantía de los derechos digitales", y con el único fin del desarrollo de esta investigación. El estudio contó con el informe favorable del Comité de Ética e Investigación del Hospital Universitario Reina Sofía de Córdoba (Código TFG-ECVPD-2019).

\section{Resultados}

Finalmente se incluyeron a 105 pacientes con una edad media de 67,8 $\pm 15,7$ años; 70 hombres $(66,7 \%)$ y 35 mujeres $(33,3 \%)$. En estadio 4 de la ERCA había 58 pacientes (55\%) y en estadio 5, 47 pacientes ( $45 \%)$.

En la Tabla 1 se representan los valores del ICC, AIVD, ABVD, Hgb, IMC y FG, del total de la muestra.

En la Tabla 2 están representados los valores para todas las dimensiones del cuestionario KDQOL-SF.

Como puede apreciarse, las dimensiones más afectadas en la CVRS fueron los Cambios en el estado de salud $(41,4 \pm 22,4)$, Situación laboral $(42 \pm 30,6)$, Carga de la enfermedad renal $(51,7 \pm 28), y$ las que mejor puntuaron fueron la Calidad de las relaciones sociales $(82 \pm 19,5)$, Función cognitiva $(81,2 \pm 21,6)$ y Función sexual $(79,3 \pm 29,1)$, de la Escala Específica para Enfermedades Renales. En cuanto al cuestionario genérico (SF-36), las dimensiones más afectadas fueron Salud general $(42,3 \pm 19,6)$, Vitalidad $(50,9 \pm 25,1)$

Tabla 1. Valores del total de la muestra.

\begin{tabular}{|l|c|c|}
\hline & Media \pm DE & IC al 95\% \\
\hline ICC (puntos) & $6,07 \pm 2,2$ & $5,62-6,48$ \\
\hline AIVD (puntos) & $6,05 \pm 2$ & $5,7-6,5$ \\
\hline ABVD (puntos) & $93,5 \pm 12$ & $91,9-96,4$ \\
\hline Hemoglobina (gr/dl) & $11,6 \pm 1,5$ & $11,3-11,9$ \\
\hline IMC (Kg/[metros]2) & $30,3 \pm 5$ & $29,0-31,5$ \\
\hline FG (ml/min/1,73m2) & $17,8 \pm 8$ & $15,94-19,28$ \\
\hline
\end{tabular}

ICC: Índice de Comorbilidad de Charlson, AIVD: Actividades Instrumentales para la Vida Diaria, ABVD: Actividades Básicas para la Vida Diaria, Hgb: Hemoglobina, IMC: Índice de Masa Corporal.
Tabla 2.Valores de la Escala Específica para Enfermedades Renales y las dimensiones del cuestionario SF-36.

\begin{tabular}{|l|c|c|}
\hline $\begin{array}{l}\text { Escalas específicas para } \\
\text { enfermedades renales }\end{array}$ & Media \pm DE & Mínimo-Máximo \\
\hline Cambios en el estado de salud & $41,4 \pm 22,4$ & $0-100$ \\
\hline Salud general & $57,9 \pm 16,9$ & $10-100$ \\
\hline Listado de síntomas/problemas & $76,6 \pm 18,3$ & $22,73-100$ \\
\hline Efectos de la enfermedad renal & $76,5 \pm 17,8$ & $25-100$ \\
\hline Carga de la enfermedad renal & $51,7 \pm 28$ & $0-100$ \\
\hline Situación laboral & $42 \pm 30,6$ & $0-100$ \\
\hline Función cognitiva & $81,2 \pm 21,6$ & $6,67-100$ \\
\hline Calidad de las relaciones sociales & $82 \pm 19,5$ & $33,33-100$ \\
\hline Función sexual & $79,3 \pm 29,1$ & $0-100$ \\
\hline Sueño & $64,3 \pm 23,8$ & $0-100$ \\
\hline Apoyo social & $76,3 \pm 27,6$ & $0-100$ \\
\hline Ítems del SF-36 & Media y DE & Mínimo-Máximo \\
\hline Función física & $58,7 \pm 30,9$ & $0-100$ \\
\hline Rol físico & $52,4 \pm 42,4$ & $0-100$ \\
\hline Dolor & $64,1 \pm 30,7$ & $0-100$ \\
\hline Salud general & $42,3 \pm 19,6$ & $5-90$ \\
\hline Bienestar emocional & $67,3 \pm 21,7$ & $16-100$ \\
\hline Rol emocional & $73,5 \pm 38,8$ & $0-100$ \\
\hline Función social & $73,3 \pm 28,6$ & $0-100$ \\
\hline Vitalidad & $50,9 \pm 25,1$ & $0-100$ \\
\hline & & \\
\hline
\end{tabular}

y Rol físico $(52,4 \pm 42,4)$; y las que mejor puntuaron fueron el Rol emocional $(73,5 \pm 38,8)$, Función social $(73,3 \pm 28,6)$ y Bienestar emocional, $(67,3 \pm 21,7)$.

Para comparar los resultados del cuestionario SF-36 de la muestra, respecto a la población general, se utilizaron los valores poblacionales para personas de la misma edad, pudiéndose observar que todas las dimensiones de este cuestionario están disminuidas respecto a la población sana de referencia, siendo las diferencias más acusadas en las dimensiones Función física (diferencia de 20,2 puntos) y Rol físico (diferencia de 34,6 puntos) (Figura 1).

Al relacionar la edad con las dimensiones de ambos cuestionarios, sólo se encontró relación estadísticamente significativa con las dimensiones Función física $(r=-0,458, p<0,01)$ y Dolor $(r=0,213, p<0,05)$ del cuestionario SF-36. También se encontró relación entre la edad y el ICC $(r=0,574, p<0,01)$.

Para el grupo de pacientes de menor aclaramiento renal (estadio 5), la media de edad fue de $71,6 \pm 12,4$ 


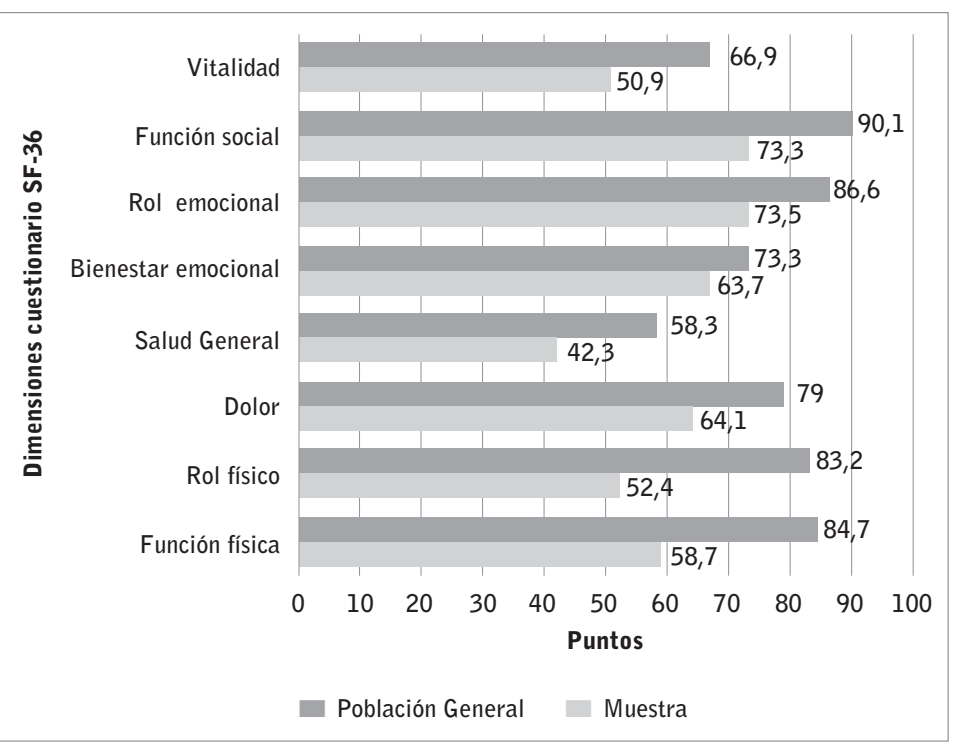

Figura 1. Valores medios de las dimensiones de la muestra estudiada y la población española de referencia de la misma edad.

años, mientras que para el grupo con mayor aclaramiento (estadio 4) fue de $64,9 \pm 17,4$ años, siendo estas diferencias significativas $(p<0,05)$.

Al comparar las puntuaciones de la CVRS entre el grupo de pacientes en estadio 4 y estadio 5, se observaron peores puntuaciones en general en los pacientes con peor función renal (estadio 5), aunque las diferencias no fueron estadísticamente significativas (Tabla 3).

Respecto a la influencia del sexo en la CVRS, no se encontraron diferencias estadísticamente significativas entre hombres y mujeres para el cuestionario específico de enfermedades renales (KDQOL), excepto para la Función sexual $(73,8 \pm 30$ frente a $91,8 \pm 22$, $p<0,01)$. Para el cuestionario genérico SF-36, no se encontraron diferencias significativas entre hombres y mujeres en ninguna de sus dimensiones.

EI ICC se correlacionó de forma significativa con la dimensión Situación laboral $(r=-0,245, p<0,05)$ del cuestionario KDQ0L y con la Función física $(r=-0,397, p<0,01)$, Salud general $(r=-0,222, p<0,05)$ y Vitalidad $(r=-$
$0,207, p<0,05)$ del SF-36. También el ICC se relacionó estadísticamente con las AIVD ( $r=-$ $0,281, p<0,01)$ y con las ABVD $(r=-0,293$, $p<0,01)$. No se encontraron relaciones estadísticas ni con los niveles de Hgb, el FG ni con el IMC.

El $57,1 \%$ de la muestra tenían algún grado de dependencia para las AIVD (Figura 2) y un $33,3 \%$ de dependencia para las ABVD.

Para comparar la CVRS entre los pacientes con mejores puntuaciones frente a las peores para las AIVD, se dividió a los pacientes en dos grupos: Dependientes (total, severa, moderada, puntuaciones 0-5) e Independientes (leve, independencia, puntuaciones 6-8), cuyos resultaos pueden observarse en la Tabla 4.

Como puede observarse, los pacientes Dependientes puntuaron peor en todas las dimensiones de la CVRS, con diferencias estadísticamente significativas en las dimensiones Efectos de la enfermedad renal, Situación laboral, Función cognitiva y Sueño, del cuestionario KDQOL; y en

Tabla 3.Comparación entre pacientes en estadio 4 y pacientes en estadio 5, para las dimensiones del cuestionario KDQOL-SF.

\begin{tabular}{|l|c|c|c|}
\hline $\begin{array}{l}\text { Escalas específicas } \\
\text { para enfermedades renales }\end{array}$ & Estadio 4 & Estadio 5 & Valor de $\mathbf{p}$ \\
\hline Listado de síntomas/problemas & $77 \pm 18,7$ & $76 \pm 18$ & NS \\
\hline Efectos de la enfermedad renal & $77,5 \pm 16,8$ & $75,2 \pm 19,1$ & NS \\
\hline Carga de la enfermedad renal & $52,7 \pm 29$ & $50,4 \pm 27$ & NS \\
\hline Situación laboral & $44,6 \pm 34$ & $38,9 \pm 25,9$ & NS \\
\hline Función cognitiva & $82,8 \pm 20,7$ & $79,3 \pm 22,7$ & NS \\
\hline Calidad de las relaciones sociales & $82,4 \pm 21$ & $81,6 \pm 17,8$ & NS \\
\hline Función sexual & $82,5 \pm 24,9$ & $74,6 \pm 34,2$ & NS \\
\hline Sueño & $66,8 \pm 25,5$ & $61,2 \pm 21,3$ & NS \\
\hline Apoyo social & $80,5 \pm 26$ & $71,3 \pm 29$ & NS \\
\hline Ítems del SF-36 & $\mathbf{E s t a d i o} 4$ & $\mathbf{E s t a d i 0} 5$ & Valor de $\mathbf{p}$ \\
\hline Función física & $61 \pm 31$ & $55,9 \pm 30,9$ & NS \\
\hline Rol físico & $56 \pm 42,5$ & $47,9 \pm 42,3$ & NS \\
\hline Dolor & $63,9 \pm 32,2$ & $64,5 \pm 29,1$ & NS \\
\hline Salud general & $43,9 \pm 20,2$ & $40,4 \pm 18,7$ & NS \\
\hline Bienestar emocional & $67,5 \pm 22,3$ & $67 \pm 21,2$ & NS \\
\hline Rol emocional & $74,4 \pm 38$ & $72,3 \pm 40,1$ & NS \\
\hline Función social & $75,2 \pm 31$ & $71 \pm 25,6$ & NS \\
\hline Vitalidad & $51,4 \pm 26$ & $50,3 \pm 24,3$ & NS \\
\hline
\end{tabular}




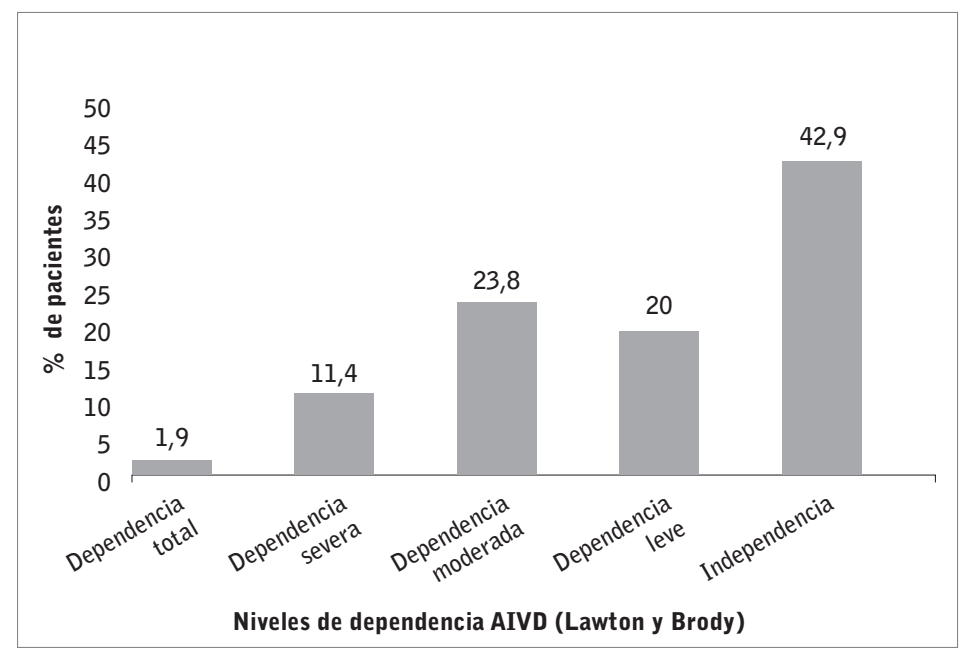

Figura 2. Porcentaje de pacientes según el nivel de dependencia para actividades instrumentales de la vida diaria.
Cuando se analizó la dependencia para las $A B V D$, se encontró que no había pacientes incluidos en las categorías de dependencia Total ni Grave, un 3,8\% presentaban dependencia Moderada, un 29,5\% dependencia Leve y el $66,7 \%$ eran Independientes. No se encontraron diferencias entre hombres y mujeres $(94,3 \pm 11$ frente a $91,8 \pm 14$ puntos, NS) ni entre pacientes en estadio 4 y estadio $5(92 \pm 12$ frente a $94,7 \pm 12$ puntos, NS), para las ABVD. Se encontró una buena correlación entre las AIVD y las ABVD $(r=0,684, p<0,01)$.

Se encontró además una correlación entre la Hgb y la dimensión Función física del SF-36 $(r=0,216, p<0,01)$.
Tabla 4. Comparación entre pacientes dependientes e independientes para las AIVD, para las dimensiones de los cuestionarios KDQOL-SF.

\begin{tabular}{|c|c|c|c|}
\hline $\begin{array}{l}\text { Escalas específicas para } \\
\text { enfermedades renales }\end{array}$ & $\begin{array}{l}\text { Dependencia } \\
\text { (Lawton 0-5) }\end{array}$ & $\begin{array}{l}\text { Independencia } \\
\text { (Lawton 6-8) }\end{array}$ & Valor de $\mathbf{p}$ \\
\hline Listado de síntomas/problemas & $72,6 \pm 20,7$ & $78,9 \pm 16,4$ & NS \\
\hline Efectos de la enfermedad renal & $70,9 \pm 23,2$ & $79,7 \pm 12,8$ & $p<0,05$ \\
\hline Carga de la enfermedad renal & $45,8 \pm 31,8$ & $55,1 \pm 25,2$ & NS \\
\hline Situación laboral & $33,8 \pm 23,7$ & $46,9 \pm 33,2$ & $p<0,05$ \\
\hline Función cognitiva & $74,7 \pm 26,2$ & $85,1 \pm 17,4$ & $p<0,05$ \\
\hline Calidad de las relaciones sociales & $79,7 \pm 21,6$ & $83,4 \pm 18,2$ & NS \\
\hline Función sexual & $75 \pm 35,8$ & $81 \pm 26,1$ & NS \\
\hline Sueño & $58,3 \pm 24,3$ & $67,9 \pm 22,9$ & $p<0,05$ \\
\hline Apoyo social & $72,2 \pm 30,2$ & $78,8 \pm 25,9$ & NS \\
\hline Ítems del SF-36 & Dependencia & Independencia & Valor de $\mathbf{p}$ \\
\hline Función física & $41,2 \pm 31,7$ & $69,1 \pm 25,3$ & $p<0,01$ \\
\hline Rol físico & $40,4 \pm 41,6$ & $59,5 \pm 41,5$ & $p<0,05$ \\
\hline Dolor & $53,3 \pm 30,5$ & $70,6 \pm 29,2$ & $p<0,01$ \\
\hline Salud general & $41,2 \pm 19,8$ & $43 \pm 19,6$ & NS \\
\hline Bienestar emocional & $66,6 \pm 21,6$ & $67,8 \pm 21,9$ & NS \\
\hline Rol emocional & $73,5 \pm 39,9$ & $73,5 \pm 38,5$ & NS \\
\hline Función social & $63,1 \pm 31,5$ & $79,4 \pm 25,1$ & $p<0,01$ \\
\hline
\end{tabular}

\section{Discusión}

La ERCA es una enfermedad que, especialmente en los estadios 4-5, impone una serie de limitaciones a la persona que la padece, incidiendo negativamente en su calidad de vida ${ }^{7}$. Sin embargo, no es una variable muy estudiada en esta fase de la enfermedad renal ${ }^{10}$; aunque algunos autores han destacado que la CVRS es un factor de riesgo relevante en la progresión de la $\mathrm{ERCA}^{18}$.

Con respecto a las dimensiones de las Escalas Específicas para Enfermedades Renales del cuestionario KDQOL, las más afectadas fueron la Carga de la enfermedad renal, la Situación laboral y el Sueño. Mientras que las que obtuvieron mejor puntuación fueron la Función cognitiva, la Calidad de las relaciones sociales y la Función sexual; resultados si-

las dimensiones Función física, Rol físico, Dolor, Función social y Vitalidad, del cuestionario SF-36.

No existieron diferencias entre hombres y mujeres para las AIVD (5,8 \pm 2 frente a $6,4 \pm 2$ puntos, NS). Tampoco se encontraron diferencias entre los pacientes en estadio 4 y estadio 5 para las AIVD $(5,8 \pm 2$ frente a $6,2 \pm 2$ puntos, NS). milares a los encontrados por otros autores ${ }^{17}$. Hay que señalar que la baja puntuación en la situación laboral no es valorable, puesto que gran parte de la muestra se encuentra en edad de jubilación.

En cuanto al cuestionario SF-36, las dimensiones más afectadas fueron la Salud general, la Vitalidad y el Rol físico; y las mejor conservadas fueron el Rol emocional 
y la Función social, en la línea de los publicado por otros autores ${ }^{16,19}$. Tal y como puede apreciarse en la Figura 1, la muestra presenta peor CVRS en todas las dimensiones si la comparamos con la población general de su misma edad.

Cuando se comparó la CVRS entre los pacientes según su FG, los pacientes en estadio 5, es decir, con peor función renal, presentaron peores puntuaciones en casi todas dimensiones, tanto en el cuestionario específico de la enfermedad renal, como en el genérico de calidad de vida, aunque estas diferencias no fueron estadísticamente significativas, posiblemente por no ser muy amplia la muestra en cada uno de los estadios. Estos resultados contrastan con los de otros autores que sí encontraron peor CVRS en el estadio 5 de la enfermedad ${ }^{17,20}$. Sin embargo, en otro estudio encontraron peores puntuaciones en el estadio 4, aunque sin diferencias significativas ${ }^{21}$. Estos autores sugieren que la falta de relación significativa entre FG y CVRS podría deberse a que los síntomas que acompañan a la enfermedad renal empeoran dicha calidad, más que la enfermedad por sí misma, y añaden que algunas causas de la ERCA pueden tener gran impacto en la calidad de vida independientemente de los efectos negativos sobre la función renal ${ }^{21}$.

Por otro lado, en esta muestra se observa que a mayor edad peor Función Física y más comorbilidad, lo cual ha sido observado en otros estudios ${ }^{16,17,22}$. La comorbilidad, además, influye directamente en varias dimensiones de la CVRS, a mayor comorbilidad, peores puntuaciones en la Situación laboral, la Función física, la Salud general y la Vitalidad. De igual forma, mayor comorbilidad se relaciona con mayor dependencia para las AIVD y ABVD; condicionando todo ello peor calidad de vida, tal como destacan otros autores ${ }^{23-25}$.

En cuanto al sexo, a pesar de que en la mayoría de estudios $^{23}$ se refleja peor calidad de vida en el sexo femenino, en nuestra muestra no hubo diferencias entre hombres y mujeres; excepto para la Función sexual, donde las mujeres presentan mayor puntuación que los hombres, en la línea de lo publicado en otros estudios, tanto en prediálisis ${ }^{16}$ como en hemodiálisis ${ }^{26}$. Esto puede deberse a que, en nuestro estudio, la muestra de mujeres es pequeña.

Cuando se analizó la dependencia, un 57,1\% de la muestra presentaba algún grado de dependencia para las AIVD y un 33,3\% para las ABVD. En estudios realizados en pacientes con ERCA en TRS con hemodiá- lisis, también obtuvieron un mayor porcentaje de dependencia para las AIVD que para las ABVD ${ }^{13,27}$. Estos resultados son coherentes con la bibliografía existente al respecto pues las AIVD son actividades más complejas, con mayor requerimiento de autonomía personal e interacción con el medio, y que aparecen más precozmente que los déficits en las $A B V D^{12}$. Aunque existe una buena correlación entre las AIVD y las $A B V D$, es evidente que son instrumentos diferentes y no tienen el mismo significado en cuanto a la detección de dependencia, ya que para las AIVD el $42,9 \%$ de la muestra era independiente, mientras que para las ABVD lo era el $66,7 \%$. No obstante, lo importante es la detección de algún grado de dependencia en estos pacientes con alta comorbilidad, pues como han destacado algunos autores existe relación entre dependencia y mortalidad temprana ${ }^{28,29}$.

Al dividir los pacientes en dependientes e independientes para las AIVD para analizar su influencia en la CVRS, se observó que los pacientes incluidos en el grupo de dependientes (dependencia total, severa y moderada) presentaban peores puntuaciones con diferencias significativas en las dimensiones Efectos de la enfermedad renal, Situación laboral, Función cognitiva y Sueño, del cuestionario KDQOL; y en las dimensiones Función física, Rol físico, Dolor, Función social y Vitalidad, del cuestionario SF-36. Esto indica, como cabe esperar, que la dependencia para las AIVD empeora la CVRS. En este sentido, no hemos encontrado bibliografía al respecto en pacientes en prediálisis, pero Arenas MD et al.13 en su estudio con pacientes en hemodiálisis, muestran resultados muy parecidos a los de nuestro estudio, aunque los pacientes no estén todavía en TRS.

En nuestra muestra los niveles de $\mathrm{Hgb}$ no parecen ser tan influyente como en otros estudios, pues tan solo encontramos relación estadística con peores puntuaciones en la dimensión Función física. Sin embargo, otros autores han relacionado niveles bajos de $\mathrm{Hgb}$ con peores puntuaciones en la CVRS en general ${ }^{30}$.

Aunque no existe mucha bibliografía en pacientes con ERCA en estadio 4-5 o prediálisis, que analicen los mismos objetivos que este trabajo, en estudios en pacientes que ya han precisado TRS, han encontrado resultados muy similares, como que la edad avanza$\mathrm{da}$, la comorbilidad y el grado de dependencia influyen en la peor percepción de la CVRS ${ }^{30}$, con altos grados de dependencia; por lo que la detección temprana de la misma, evaluando periódicamente a los pacientes y 
remitiéndolos a programas de rehabilitación (física, cognitiva, psicológica, ambiental...), puede mejorar la función en las AIVD ${ }^{31}$. Por todo ello, creemos que es importante actuar cuanto antes, en la etapa de prediálisis, donde ya se comienzan a ver resultados parecidos a los de los pacientes en TRS, para retrasar ese desgaste producido por la enfermedad y mejorar la CVRS.

Como principales limitaciones hay que destacar que la muestra es pequeña y dentro de esta el número de mujeres es escaso. Otra limitación es la falta de referencias bibliográficas que analicen los mismos objetivos que nuestro estudio. Otra limitación puede ser un sesgo de género que ha podido influenciar las puntuaciones del Test de Lawton y Brody, ya que algunos de sus ítems se atribuyen socioculturalmente a la mujer (ir de compras, preparación de la comida, cuidado de la casa y lavado de la ropa).

A la vista de estos resultados podemos concluir que al menos, en la muestra estudiada, los pacientes con ERCA en estadios 4-5, tienen disminuida su CVRS, con afectación en la mayoría de las dimensiones que engloba este concepto, en comparación con la población sana de referencia, siendo las dimensiones más afectadas los Cambios en el estado de salud, Carga de la enfermedad renal, Salud general, Vitalidad y Rol físico.

Más de la mitad de los pacientes presenta algún grado de dependencia para las AIVD influyendo esto negativamente en su CVRS, en comparación con el grupo de pacientes que no presenta dependencia.

Además de la dependencia funcional, otras variables como la comorbilidad y la edad, son factores influyentes en la peor CVRS de estos pacientes.

Por ello y por la falta de estudios en esta etapa de la enfermedad, creemos importante seguir investigando en esta línea y promover la actuación de Enfermería desde las consultas de ERCA para detectar la dependencia, retrasar en lo posible la progresión de la enfermedad, y por tanto la progresión de la pérdida de la CVRS.

Recepción: 5-10-20

Aceptación: 7-11-20

Publicación: 30-12-20

\section{Bibliografía}

1. Soriano S. Definición y clasificación de los estadios de la enfermedad renal crónica: Prevalencia, claves para el diagnóstico precoz, factores de riesgo de enfermedad renal crónica. Nefrología. 2004; 24(6):27-34.

2. Alcázar R, Orte L, Otero A. Enfermedad renal crónica avanzada. Nefrología. 2008;28(3):3-6.

3. Guerrero MA, Toro FJ, Batalha $P$, Salgueira $M$, González F, Marrero S, et al. Estudio ERCA. Características basales, evaluación de la aplicación de la información estructurada para la elección de tratamiento renal sustitutivo y evolución a un año de los pacientes incidentes en la consulta ERCA. Nefrología. 2019;39(6):563-82.

4. Otero A, de Francisco A, Gayoso P, Garcia F. Prevalence of chronic renal disease in Spain: results of the EPIRCE study. Nefrología 2010;30:78-86.

5. Registro Español de Enfermos Renales. Informe de diálisis y trasplante 2018. [consultado 30 marzo 2019]. Disponible en: https://www.senefro.org/contents/webstructure/SEN_2019_REER_modificada. pdf.

6. Górriz JL, Otero A. Impacto socio sanitario de la enfermedad renal crónica avanzada. Nefrología. 2008;28(3):7-15.

7. Rebollo-Rubio A, Morales-Asencio JM, Pons-Raventos ME, Mansilla M. Revisión de estudios sobre calidad de vida relacionada con la salud en la enfermedad renal crónica avanzada en España. Nefrología. 2015;35(1):92-109.

8. García-Llana H, Remor E, Peso G, Selgas R. El papel de la depresión, la ansiedad, el estrés y la adhesión al tratamiento en la calidad de vida relacionada con la salud en pacientes en diálisis: revisión sistemática de la literatura. Nefrología. 2014; 34(5):637-57.

9. Gutiérrez D, Leiva-Santos JP, Sánchez-Hernández R, Gómez R. Prevalencia y evaluación de síntomas en enfermedad renal crónica avanzada. Enferm Nefrol. 2015;18(3):228-36.

10. Sanz-Guajardo D, Orte L, Gómez-Campderá F, Fernández E, Aguilar MD, Lázaro P. Calidad de vida en los pacientes con insuficiencia renal crónica. Influencia de la intervención precoz del nefrólogo y de 
la consulta prediálisis. Nefrología. 2006;26 (3):5665.

11. Hernández K, Neumann V. Análisis de instrumento para evaluación del desempeño en actividades de la vida diaria instrumentales Lawton y Brody. Revista Chilena de Terapia Ocupacional. 2016;16(2):55-62.

12. Kavanagh NT, Schiller B, Saxena AB, Thomas IC, Kurella M. Prevalence and correlates of functional dependence among maintenance dialysis patients. Hemodial Int. 2015;19(4):593-600.

13. Arenas MD, Navarro M, Serrano E, Álvarez-Ude F. Dependencia para las actividades instrumentales de la vida diaria en pacientes en hemodiálisis: influencia sobre la calidad de vida relacionada con la salud. Nefrología. 2019;39(5):531-38.

14. Rebollo A, Morales JM, Pons $M^{a}$ E. Influencia de la consulta de enfermería de enfermedad renal crónica avanzada en pacientes que inician tratamiento renal sustitutivo. Enferm Nefrol. 2014;17(4):243-50.

15. Forero J, Barrios S. Rol de enfermería en la consulta de prediálisis en el paciente con enfermedad renal crónica avanzada. Enferm Nefrol. 2016;19 (1):77-86.

16. Ruiz-Parrado MC, Gómez-Pozo M, Crespo M, Tierno C, Crespo-Montero R. Análisis de la calidad de vida del paciente en la etapa prediálisis. Enferm Nefrol. 2017;20(3):233-40.

17. Garrido-Blanco R, Arroyo-Priego E, Arana-Ruiz AI, López-Zamorano MD, Tierno-Tendero C, Crespo-Montero R. Calidad de vida y enfermedad renal crónica avanzada. Influencia del aclaramiento renal. Enferm Nefrol. 2018;21(4):359-67.

18. Oh TR, Choi HS, Kim CS, Bae EH, Oh YK, Kim Y-S, et al. Association between health related quality of life and progression of chronic kidney disease. Sci Rep 9, 19595 (2019).

19. Cruz MC, Andrade $C$, Urrutia M, Draibe $S$, Nogueira-Martins LA, de Castro Cintra Sesso R. Quality of life in patients with chronic kidney disease. Clinics (Sao Paulo). 2011;66(6):991-5.

20. Pagels AA, Söderkvist B, Medin C, Hylander B, Heiwe $S$. Health-related quality of life in different stages of chronic kidney disease and at initiation of dialysis treatment. Health Qual Life Outcomes. 2012;10:71.
21. Perlman RL, Finkelstein F0, Liu L, Roys E, Kiser $M$, Eisele $G$, et al. Quality of life in Chronic Kidney Disease (CKD): A cross-sectional analysis in the Renal Research Institute-CKD study. Am J Kidney Dis. 2005;45(4):658-66.

22. Yepes $C E$, Montoya $M$, Orrego BE, Cuéllar MH, Yepes JJ, López JP, Salguero MV, Gómez RD. Calidad de vida en pacientes con enfermedad renal crónica sin diálisis ni trasplante de una muestra aleatoria de dos aseguradoras en salud. Medellín, Colombia, 2008. Nefrología. 2009;29(6):548-56.

23. Alhaji M, Tan J, Abdul Hamid M, Timbuak J, Naing $\mathrm{L}$, Tuah N. Determinants of quality of life as measured with variants of SF-36 in patients with predialysis chronic kidney disease. Saudi Med J. 2018;39(7):653-61.

24. Román $R$, Cereto $R$, Reyes $M$, Alférez $A$, Merino P. Calidad de Vida Relacionada con la Salud en las diferentes terapias sustitutivas de la Insuficiencia Renal Crónica. Rev Soc Esp Enferm Nefrol 2003; 6(4):6-16.

25. Albañil T, Ramírez MC, Crespo R. Análisis de la calidad de vida en pacientes en hemodiálisis ambulatoria y su relación con el nivel de dependencia. Enferm Nefrol. 2014;17(3):167-74.

26. Lleixà MA, Marin LC, Solé JA, Miravete ES, Martínez E, Usach TS. Valoración de la calidad de vida relacionada con la salud en la enfermedad renal crónica terminal mediante un cuestionario de resultados percibidos por los pacientes. Enferm Nefrol. 2016;19(4):331-40.

27. Camps $E_{\text {, Andreu }} \mathrm{L}$, Colomer $\mathrm{M}$, Claramunt $\mathrm{L}$, Pasaron M. Valoración del grado de autonomía funcional de pacientes renales crónicos según índices de Barthel, Lawton y baremo de Ley de Dependencia. Rev Soc Esp Enferm Nefrol. 2009;12(2):104-10.

28. Andreu-Periz L, Puig-Llobet M, Cases-Amenós A. Grado de dependencia de las personas sometidas a hemodiálisis en Cataluña y evolución de su mortalidad. Nefrología. 2012;32(5):613-21.

29. Jassal SV, Karaboyas A, Comment LA, Bieber BA, Morgenstern $H$, Sen $A$, et al. Functional Dependence and Mortality in the International Dialysis Outcomes and Practice Patterns Study (DOPPS). Am J Kidney Dis. 2016;67(2):283-92.

30. Kefale B, Alebachew M, Tadesse Y, Engidawork E. Quality of life and its predictors among patients 
with chronic kidney disease: A hospital-based cross sectional study. Plos One. 2019. Febrero(27).

31. Sánchez-Cabezas AM, Morillo-Gallego N, Merino-Martínez RM, Crespo-Montero R. Calidad de vida de los pacientes en diálisis. Revisión sistemática. Enferm Nefrol. 2019;22(3):239-55.

32. Farragher J, Jassal SV. Rehabilitation of the Geriatric Dialysis Patient: Rehabilitation of the geriatric dialysis patient. Semin Dial. 2012;25(6):649-56.

Este artículo se distribuye bajo una Licencia Creative Commons Atribución-NoComercial 4.0 Internacional. https://creativecommons.org/licenses/by-nc/4.0/

\section{Open Access (c) () \&}

